\title{
Banda de Histéresis Adaptativa para un Convertidor AC-DC Elevador sin Puente, con Correccion del Factor de Potencia y Control por Modos Deslizantes
}

\author{
Gabriel E. Mejía-Ruiz(1), Nicolás Muñoz-Galeano(2), y José R. Ortiz-Castrillón(2) \\ (1) Grupo de investigación I2, Facultad de Ingeniería, Corporación Universitaria Minuto de Dios UNIMINUTO, Carrera 45 \\ No. 22D - 25, Bello - Colombia (e-mail: gabriel.mejia@uniminuto.edu) \\ (2) Grupo de Investigación GIMEL, Departamento de Ingeniería Eléctrica, Facultad de Ingeniería, Universidad de \\ Antioquia, Calle 67 No.53-108, Medellín - Colombia (e-mail: nicolas.munoz@udea.edu.co; jrobinson.ortiz@udea.edu.co)
}

Recibido Nov. 20, 2018; Aceptado Ene. 21, 2019; Versión final Feb. 22, 2019, Publicado Ago. 2019

\begin{abstract}
Resumen
Se propone aplicar una banda de histéresis adaptativa que permite reducir la distorsión armónica total, el rizado y la deformación en el cruce por cero de la corriente de entrada de un convertidor AC-DC elevador sin puente con corrección del factor de potencia (PFC, Power Factor Correction). Se presenta un procedimiento para calcular la banda de histéresis con base en los estados dinámicos del convertidor electrónico de potencia, de tal forma que es posible fijar la frecuencia de conmutación. La banda es aplicable al control por modos deslizantes que permite mejorar el desempeño dinámico de los convertidores ante perturbaciones acotadas y cambios significativos de la región de operación. Se presentan los resultados de la simulación de un convertidor de $1 \mathrm{~kW}$. Se muestra que es posible elevar el factor de potencia hasta 0.996 , disminuir la deformación del cruce por cero, y reducir la distorsión armónica total en la corriente de entrada hasta $2.8 \%$, manteniéndola por debajo de los límites máximos permisibles del estándar EN/IEC 61000-3-2 (1000-3-2).
\end{abstract}

Palabras clave: banda de histéresis adaptativa; corrector del factor de potencia; rectificador controlado; modos de deslizamiento; frecuencia constante

\section{Adaptive Hysteresis Band for a Bridgeless Boost Converter with Power Factor Correction and Sliding Mode Control}

\begin{abstract}
It is proposed to apply an adaptive hysteresis band that allows to reduce the total harmonic distortion, the ripple and the deformation in the zero crossing of the input current of a bridgeless boost AC-DC converter with power factor correction (PFC). A procedure for calculating the hysteresis band based on the dynamic states of the converter is presented, in such a way that it is possible to keep fixed the switching frequency. The band is applicable to the sliding mode control that allows to improve the dynamic performance of the power converter in the presence of bounded perturbations and significant changes in operating region. The results of the simulation of a $1 \mathrm{~kW}$ converter are presented. It is shown that it is possible to raise power factor up to 0.996 , to decrease the deformation of the zero crossing and to reduce the total harmonic distortion in the input current down to $2.8 \%$, keeping it below the maximum permissible limits of the standard EN / IEC 61000-3-2 (1000-32).
\end{abstract}

Keywords: adaptive hysteresis band; power factor corrector; controlled rectifier; sliding mode; constant frequency 


\section{INTRODUCCIÓN}

El convertidor AC-DC elevador sin puente (SBLB PFC, Semi Bridgeless Boost Power Factor Correction) presenta oscilaciones de amplitud y frecuencia finita alrededor de la señal de referencia (Muñoz-Galeano et al., 2016); este fenómeno, llamado "rizado" en la literatura de electrónica de potencia o "chattering" en la teoría de control reduce la precisión del controlador, incrementa las pérdidas de potencia y aumenta la distorsión armónica total en la corriente de entrada (THDi; Total Harmonic Distortion in input current) (Utkin et al., 2017; Young et al., 1999). El control lineal con modulación PWM puede: reducir el rizado y el THDi, aumentar el factor de potencia (PF, Power Factor) (Mejía-Ruiz et al., 2017; Huang et al., 2017; Kim et al., 2014), reducir el ruido de modo común (Man et al., 2017), incrementar la eficiencia (Alam et al., 2017; Bin y Lu, 2015; ChihChiang et al., 2016) y reducir la cantidad de sensores necesarios para el control de los convertidores electrónicos de potencia (Chen et al., 2017; Musavi et al., 2013). No obstante, el diseño de este controlador se realiza con base en el modelo lineal del convertidor y parámetros constantes; además, el cambio en la región de operación del convertidor y la variación de los parámetros limita la regulación y la respuesta dinámica del control lineal, estando inhabilitado para compensar perturbaciones significativas en la fuente 0 en la carga (Mohanty y Panda, 2017). El sistema de control lineal también es incapaz de compensar el componente DC en la corriente cuando el SBLB PFC tiene de forma inherente inductores de características diferentes en sus terminales (Marcos-Pastor et al., 2015).

El control por modos deslizantes (SMC, Sliding Mode Control) es una técnica no lineal derivada de la teoría de los sistemas de estructura variable (VSS, Variable Structure Systems). El SMC consiste en una ley de control discontinua, variante en el tiempo y con realimentación. (Utkin, 1977; Utkin et al., 2009). El SMC permite mejorar el desempeño dinámico de los convertidores en presencia de perturbaciones acotadas, cambios de operación y variación de los parámetros. El SMC también permite reducir el orden del sistema en lazo cerrado y suministrar la señal de activación y desactivación de los actuadores sin la intervención del sistema de modulación PWM, incrementando la velocidad de respuesta del controlador en lazo cerrado (Utkin, et al., 2017; Utkin, 1977; Utkin et al., 2009; Young et al., 1999). En (Marcos-Pastor et al., 2015) se desarrolla el SMC con circuitos analógicos para el SBLB PFC usando frecuencia de conmutación variable donde se evidencia el incremento del THD debido a la deformación de la corriente en el cruce por cero; una forma de reducir esto consiste en el uso de un controlador de corriente con banda de histéresis de ancho fijo que evita la introducción del componente DC en la corriente de la fuente, asegurando la simetría en cada semi-ciclo de la red. No obstante, el control con banda de histéresis de ancho fijo causa que la frecuencia de conmutación sea variable de tal forma que se hace necesario aumentar el tamaño y el costo de los filtros, los inductores y los disipadores de calor del sistema (Huber et al., 2008). La frecuencia variable también puede producir ruido audible e incrementar las pérdidas de potencia cuando el voltaje de la fuente tiene amplitud baja y la frecuencia de conmutación es alta (Ye et al., 2014; Guzman et al., 2016; Chincholkar y Chan, 2017; Pichan y Rastegar, 2017; Repecho et al., 2017).

Varios autores reducen el rizado fijando la frecuencia de conmutación; sin embargo, las metodologías de control presentadas deterioran el desempeño dinámico y la robustez del sistema (Mohanty y Panda, 2017; Ye et al., 2014; Chincholkar y Chan, 2017; Guzman et al., 2016; Pichan y Rastegar, 2017; Ye et al., 2014; Ashita et al., 2014). Además, el sistema de control requiere alta capacidad de cómputo que usualmente incluye unidades de punto flotante y arquitecturas de memoria de acceso múltiple para realizar los cálculos necesarios para fijar la frecuencia, incrementando los costos de la implementación. En (Marcos-Pastor et al., 2016) desarrollan un controlador de corriente valle para fijar la frecuencia de conmutación ( $f_{\mathrm{sw}}$ ) del rectificador con PFC; sin embargo, este método causa el incremento del tercer armónico en la corriente de entrada. El SMC también puede usar modulación PWM para fijar la frecuencia de conmutación; pero en la práctica, esta implementación es similar al control clásico PWM basado en la ley de control lineal y requiere que la función del control equivalente sea continua, de tal forma que no es posible la reducción del orden ni mejorar la robustez del sistema (Chincholkar y Chan, 2017; Mohanty y Panda, 2017). Este artículo propone una banda de histéresis adaptativa para un convertidor AC-DC elevador sin puente con corrección automática del factor de potencia que permite fijar la frecuencia de conmutación, disminuir el rizado, reducir la deformación de la corriente en el cruce por cero y el THDi. Este artículo presenta el procedimiento de cálculo y la simulación de la banda de histéresis adaptativa mediante las siguientes secciones: el modelo dinámico de convertidor, la selección del sistema de control y el diseño detallado de la banda de histéresis propuesta. Finalmente, se presentan los resultados de la simulación y las conclusiones.

\section{DESCRIPCIÓN DEL MÉTODO DE CONTROL}

Los objetivos de control del SBLB PFC son: 1) controlar la forma de onda de la corriente de entrada (in), manteniéndola sinusoidal y en fase con el voltaje de entrada ( $\left.v_{\text {in }}\right)$ para aumentar el PF; 2 ) controlar la amplitud del rizado de $\mathrm{i}_{\mathrm{m}}$ cerca del cruce por cero para reducir el $T H \mathrm{D}_{i}$; y 3 ) regular el voltaje de salida $\left(\mathrm{V}_{0}\right)$ según los requerimientos de la carga. Además, el sistema de control deberá compensar las variaciones de la amplitud 
en $\mathrm{Vin}$ y las variaciones de la potencia en la salida $\left(\mathrm{P}_{0}\right)$. Para lograr esto, primero se obtiene el modelo dinámico del sistema a controlar, luego se selecciona el método de control y finalmente, se diseña el modulador por banda de histéresis adaptativa.

\section{Modelo dinámico del sistema a controlar con banda de histéresis adaptativa}

El circuito del SBLB PFC y el sistema de control con la banda de histéresis adaptativa propuesta en este artículo se muestran en la Fig. 1. El SBLB PFC puede descomponerse en dos convertidores elevadores operativos según el semi-ciclo de la red (Mejía-Ruiz et al., 2017), por lo que se modela el sistema para cada semiciclo. EI SBLB PFC posee dos interruptores $Q_{1}$ y $Q_{2}$, cuatro diodos $D_{1}, D_{2}, D_{3}$, y $D_{4}$, dos inductores $L_{1}$ y $\mathrm{L}_{2}$, un condensador $\mathrm{C}$ y la resistencia de carga $\mathrm{R}\llcorner$ (Alam et al., 2017; Jovanovic y Jang, 2005; Xu et al., 2014). Los interruptores $Q_{1}$ y $Q_{2}$ son accionados por la misma señal de control (u) (Franceschini et al., 2012; MarcosPastor et al., 2015) operando en la región de corte y saturación; en consecuencia, los convertidores exhiben un comportamiento dinámico no lineal y discontinuo. La obtención del modelo matemático considera las siguientes suposiciones: 1) los interruptores de potencia tienen resistencia cero en el estado de encendido y resistencia infinita en el estado de apagado, 2) el tiempo de conmutación es infinitamente corto, 3) las fuentes pueden suministrar potencia infinita en corto circuito, 4) la frecuencia de conmutación ( $f_{s w}$ ) es mucho mayor que la frecuencia de la fuente de corriente alterna $\left(f_{0}\right)\left(f_{s w} \gg f_{0}\right)$ y 5 ) los inductores $L_{1}$ y $L_{2}$ tiene igual valor $\left(\mathrm{L}_{1}=\mathrm{L}_{2}=\mathrm{L}\right)$.

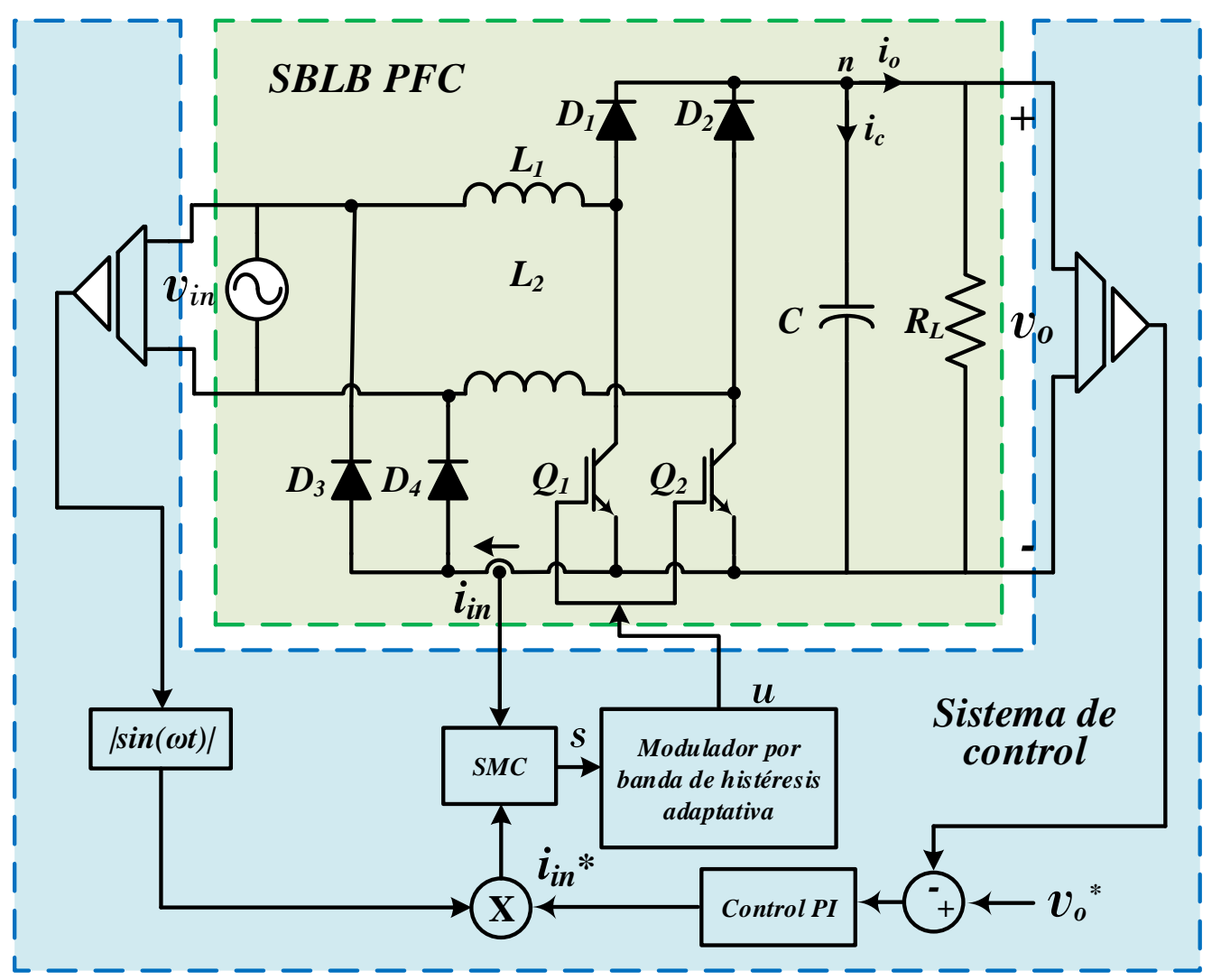

Fig. 1: Convertidor AC-DC elevador sin puente con PFC (SBLB PFC).

La banda de histéresis adaptativa se calcula con base en los estados dinámicos del SBLB PFC; razón por la cual, se encuentra el modelo del convertidor. Las ecuaciones (1) y (2) representan el modelo del sistema que es obtenido con base en las leyes de Kirchhoff y los estados de conmutación de $Q_{1}$ y Q2. U=1se establece cuando los interruptores están cerrados, mientras que $u=0$ se establece cuando los interruptores están abiertos (Mejía-Ruiz et al., 2017).

$$
\begin{aligned}
& \frac{d i_{\text {in }}}{d t}=\frac{v_{\text {in }}}{L}-\frac{v_{o}}{L}(1-u) \\
& \frac{d v_{o}}{d t}=-\frac{v_{o}}{R_{L} C}+\frac{i_{\text {in }}}{C}(1-u)
\end{aligned}
$$




\section{Selección del sistema de control}

Las dinámicas del SBLB PFC son inestables cuando se emplea solo el controlador de voltaje (Utkin, 2016). Sin embargo, esta inestabilidad se puede eliminar mediante la implementación de un sistema con dos lazos de control en cascada como se muestra en la Fig. 1 (Alsmadi et al., 2017; y Yonglu et al, 2017). Este sistema requiere que el ancho de banda del controlador interno sea al menos 10 veces mayor que el ancho de banda del controlador externo (Mejía-Ruiz et al., 2017). El lazo externo (bloque de control PI, Fig. 1) permite regular $v_{0}$ y es implementado con un controlador Proporcional Integral $(\mathrm{PI})$; los valores de la ganancia $(\mathrm{K}=0.5)$ y el tiempo de acción integral $\left(\mathrm{T}_{i}=0.3\right)$ del controlador se obtienen con base en la ubicación de los polos del sistema en lazo cerrado, teniendo como base un tiempo de establecimiento menor a $1 \mathrm{~s}$ en $\mathrm{v}_{0}$. La salida del controlador PI proporciona la amplitud de la señal de referencia para in; esta salida es multiplicada por la señal sinusoidal rectificada con la misma fase y frecuencia de $v_{\text {in }} \mathrm{y}$ el resultado es la señal de referencia ( $\mathrm{in}^{*}$ ) para el controlador interno.

El control por modos deslizantes (Sliding Mode Control, SMC) corresponde al lazo interno (bloque SMC, Fig. 1) y permite controlar la lin. El diseño del SMC comienza con la selección de la superficie de deslizamiento (S) (ecuación (3)) sobre la cual ocurre el modo de deslizamiento en el espacio de estados. El sistema en lazo cerrado se encuentra en régimen o dinámica deslizante cuando las trayectorias del sistema impuestas por la función de conmutación se dirigen hacia $S=0$. En la ecuación (3), lin=l·sen( $\omega t)$ es la corriente de entrada en estado estable. El valor de $\mathrm{I}=2\left(\mathrm{~V}_{0}{ }^{*}\right)^{2} / \mathrm{R} \mathrm{V}$ puede ser obtenido mediante un balance de potencia instantánea en la entrada y en la salida del convertidor $\left(\mathrm{P}_{\text {in }}=\mathrm{P}_{0}\right)$, donde $\left\langle\mathrm{P}_{\text {in }}\right\rangle=\mathrm{VI} / 2,\left\langle\mathrm{P}_{0}\right\rangle=\left(\mathrm{V}_{0}{ }^{*}\right)^{2} / \mathrm{R}_{\mathrm{L}}, \mathrm{V}_{0}{ }^{*}$ es el valor de referencia de $v_{0}$ y $V$ es la magnitud de $v_{\text {inn. }}$ las condiciones de alcanzabilidad, existencia, transversalidad y control equivalente también deben ser consideradas en el diseño del SMC. La condición de transversalidad evalúa la capacidad del controlador para modificar las dinámicas del sistema con la superficie de conmutación propuesta (Utkin, 1977; Utkin et al., 2009; Young et al., 1999). La ecuación (4) muestra el cumplimiento de la condición de transversalidad cuando se aplica a la superficie de conmutación definida en la ecuación (3).

La condición necesaria y suficiente para que el sistema cumpla la condición de alcanzabilidad es $S(d S / d t)<0$ para todo $t>0$ y que $|\mathrm{S}| \leq \mathrm{BH}$; es decir que, la amplitud del rizado está acotada por $\mathrm{BH}$ y los estados del sistema convergen hacia S (Alsmadi et al., 2017; Utkin, 1977; Utkin et al., 2009; y Young et al., 1999). Cuando la transversalidad es positiva, la condición de existencia puede ser determinada por la inspección local de la condición de alcanzabilidad; tal que, en el dominio de $0<|\mathrm{S}|<\mathrm{BH}$ se satisfacen las ecuaciones (5) y (6) (Alsmadi et al., 2017; Utkin, 1977; Utkin et al., 2009; Young et al., 1999) para $v_{\text {in }}>0$ y $v_{0}>V_{\text {in. }}$. El control equivalente (Ueq) es una ley de control que lleva al sistema a deslizarse sobre la superficie en forma ideal, obteniendo un valor continuo que representa el valor medio del control discontinuo; esto elimina la discontinuidad en el lado derecho de la ecuación diferencial (1) (Alsmadi et al., 2017). Ueq puede ser hallado con (dS/dt=0) como se muestra en la ecuación (7) (Alsmadi et al., 2017; Utkin, 1977; Utkin et al., 2009; Young et al., 1999). La región donde el modo de deslizamiento se produce esta dada por $0<u_{\text {eq }}<1$; es decir, el modo de deslizamiento puede producirse siempre que $v_{0}$ sea mayor que $v_{\text {in }}\left(0<v_{\text {in }}<v_{0}\right)$. La ecuación (8) representa el movimiento de los lazos de lin $y v_{0}$ durante el modo de deslizamiento que es obtenida al sustituir ueq (ecuación (10)) en las ecuaciones (1) y (2); la ecuación (8) evidencia la reducción del orden del sistema al usar SMC, ya que este permite desacoplar las dinámicas de lin y vo.

$$
\begin{aligned}
& S=i_{\text {in }}-i_{\text {in }}{ }^{*} \\
& \frac{d}{d u}\left(\frac{d S}{d t}\right)=\frac{d}{d u}\left(\frac{d i_{\text {in }}}{d t}-\frac{d i_{i n}{ }^{*}}{d t}\right)=\frac{d}{d u}\left(\frac{v_{i n}}{L}-\frac{v_{o}}{L}(1-u)\right)=\frac{v_{o}}{L} \neq 0 \\
& \left.\lim _{S=0^{-}} \frac{d S}{d t}\right|_{u=1}>0 \text {, para el SBLBPFC }\left.\lim _{S=0^{-}} \frac{d S}{d t}\right|_{u=1}=\lim _{S=0^{-}} \frac{v_{i n}}{L}>0 \\
& \left.\lim _{S=0^{+}} \frac{d S}{d t}\right|_{u=0}<0, \text { para el SBLBPFC }\left.\lim _{S=0^{+}} \frac{d S}{d t}\right|_{u=0}=\lim _{S=0^{+}}\left(\frac{v_{i n}}{L}-\frac{v_{o}}{L}\right)<0 \\
& \left.\frac{d S}{d t}\right|_{u=u_{e q}}=\frac{v_{i n}}{L}-\frac{v_{o}}{L}\left(1-u_{e q}\right)=0, \quad \text { donde } u_{e q}=1-\frac{v_{i n}}{v_{o}} \\
& \frac{d v_{o}}{d t}=-\frac{v_{o}}{R_{L} C}+\frac{2\left(v_{o}\right)^{2}}{R_{L} C v_{o}} y \frac{d i_{i n}}{d t}=0
\end{aligned}
$$




\section{Modulador por banda de histéresis adaptativa}

Este artículo propone el siguiente procedimiento de cálculo de la función del modulador por banda de histéresis adaptativa mostrado en la Fig. 1. El modulador es usado para generar la señal $u$ que acciona a $Q_{1}$ y Q2. La Fig. 2 muestra la representación geométrica de la Banda de Histéresis $(H B=\Delta \mathrm{in} / 2)$ adaptativa propuesta en términos de la amplitud del rizado $(\Delta \mathrm{i}$ in) de lin. La banda adaptativa permite la operación con $f_{s w}=1 / T_{s w}=1 /\left(T_{\text {on }}+T_{\text {off }}\right)$ constante, donde $T_{\text {sw }}$ es el periodo de conmutación, $T_{\text {on }} y T_{\text {off }}$ son el tiempo de apertura y de cierre de los interruptores respectivamente.

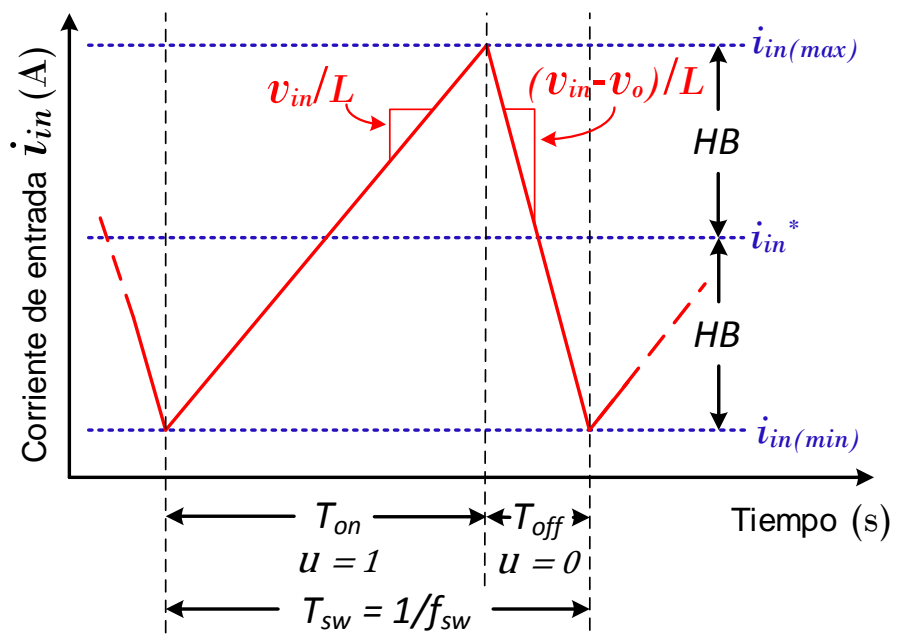

Fig. 2: Representación geométrica de la banda de histéresis adaptativa.

En el cálculo de HB, se asume que los cambios de $i_{\text {in }}$ respecto al tiempo son lineales $(\mathrm{din} / \mathrm{dt}=\Delta \mathrm{i}$ in $/ \Delta \mathrm{t}$ ). Durante la operación del SBLB PFC, $Q_{1}$ y Q $Q_{2}$ están cerrados incrementando la magnitud de lin hasta alcanzar el límite superior (in(max)) de HB; por otro lado, lin está decreciendo cuando $Q_{1}$ y $Q_{2}$ están abiertos mientras se alcanza el límite inferior ( $\operatorname{lin(\operatorname {min}))}$ de HB. La ecuación (9) se obtiene al reemplazar $u=1$ en la ecuación (1), donde $V_{\text {in }}=\mathrm{V}|\sin (\omega t)|$ debido a la acción rectificadora del convertidor, $\mathrm{v}_{0}$ es el voltaje regulado en la salida y $\mathrm{V}$ es la amplitud de vin. La ecuación (10) se obtiene al reemplazar u=0 (interruptores abiertos) en la ecuación (1).

$$
\begin{aligned}
& \frac{\Delta i_{\text {in }}}{\Delta t} \cong \frac{v_{\text {in }}}{L}=\frac{\Delta i_{\text {in }}}{T_{\text {on }}}, \text { donde } T_{\text {on }}=L \frac{\Delta i_{\text {in }}}{v_{\text {in }}} \\
& \frac{\Delta i_{\text {in }}}{\Delta t} \cong \frac{v_{0}-v_{\text {in }}}{L}=\frac{\Delta i_{\text {in }}}{T_{\text {off }}} \text {, donde } T_{\text {off }}=L \frac{\Delta i_{\text {in }}}{v_{0}-v_{\text {in }}}
\end{aligned}
$$

La ecuación (11) se obtiene al sustituir $T_{\text {on }}$ (ecuación 9) y $T_{\text {off }}$ (ecuación (10)) en f $\mathrm{sw}_{\mathrm{sw}}=1 /\left(\mathrm{T}_{\mathrm{on}}+\mathrm{T}_{\text {off }}\right)$.

$$
\Delta i_{i n}=\frac{v_{i n}\left(v_{o}-v_{i n}\right)}{L f_{s w} v_{o}}
$$

La ecuación (12) presenta la banda de histéresis adaptativa propuesta en este artículo que puede ser obtenida reemplazando la ecuación (11) en $\mathrm{HB}=\Delta \mathrm{i}$ in/2.

$$
H B=\frac{\Delta i_{i n}}{2}=\frac{v_{i n}\left(v_{o}-v_{i n}\right)}{2 L f_{s w} v_{o}}=\frac{E|\sin (\omega t)|\left(v_{o}-E|\sin (\omega t)|\right)}{2 L f_{s w} v_{o}}
$$

El diagrama de bloques del modulador por histéresis con $f_{s w}$ constante se muestra en la Fig. 3. La amplitud de HB se calcula con base en la ecuación (12) y los valores de $\mathrm{V}_{0}$, Vin, $\mathrm{f}_{\mathrm{sw}} \mathrm{y} \mathrm{L}$. La señal resultante del cálculo es comparada con $S$ y el resultado de la comparación es aplicado a las entradas de un flip-flop RS. Q1 y $Q_{2}$ se abren cuando $S$ es mayor que HB y se cierran cuando $S$ es menor que HB, limitando la amplitud del rizado de lin con la banda de histéresis adaptativa. La implementación de la banda de histéresis propuesta puede ser extendida a sistemas trifásicos; esto, debido a que la corriente de cada fase puede ser controlada siguiendo su propia banda de histéresis, teniendo en cuenta que la referencia a seguir por el control se construye con base en la señal de voltaje de su propia fase. 


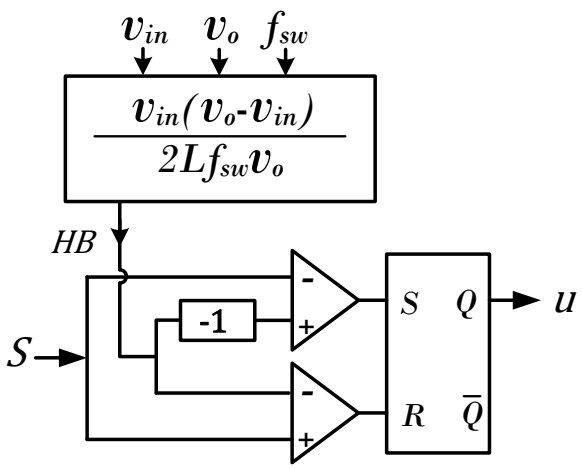

Fig. 3: Diagrama de bloques del modulador con banda de histéresis adaptativa.

\section{RESULTADOS}

El circuito SBLB PFC fue simulado en el software PSIM. La topología y el esquema de control se muestran en la Fig. 4. Los valores de los componentes y las especificaciones funcionales usados en la simulación del SBLB PFC se presentan en la Tabla 1. El objetivo de la simulación consiste en evaluar el desempeño de la banda de histéresis adaptativa propuesta en este artículo; por lo tanto, se presenta un análisis de las formas de onda de la corriente y el THDi obtenido.

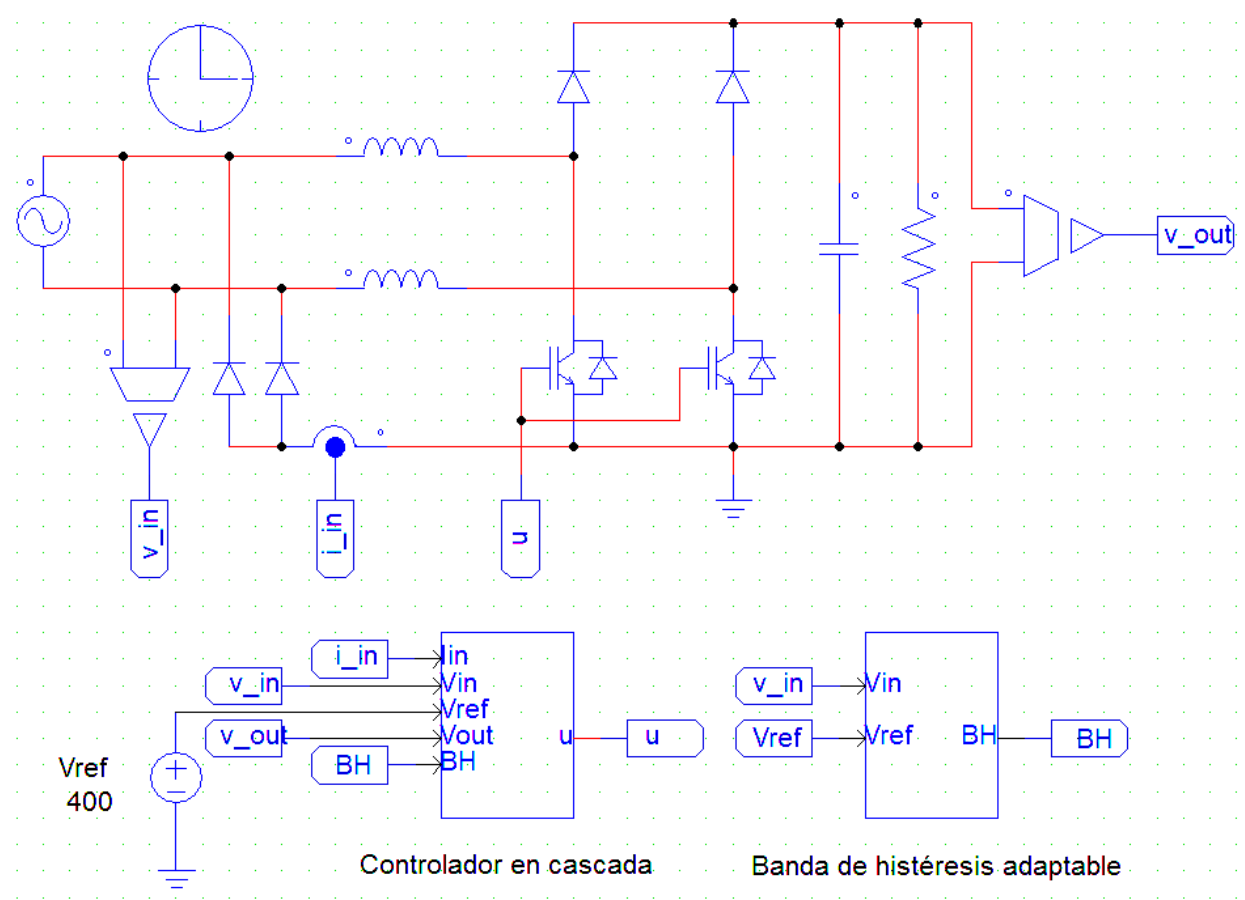

Fig. 4: Circuito del SBLB PFC simulado en PSIM.

Tabla 1: Especificaciones funcionales del SBLB PFC.

\begin{tabular}{|c|l|c|}
\hline Símbolo & \multicolumn{1}{|c|}{ Descripción } & Valor \\
\hline$V_{\text {in }}$ & Voltaje de entrada & $84 \mathrm{~V}_{\text {rms }}$ hasta $120 \mathrm{~V}_{\mathrm{rms}}$ \\
\hline $\mathrm{f}_{\mathrm{L}}$ & Frecuencia de la línea & $60 \mathrm{~Hz}$ \\
\hline $\mathrm{V}_{0}$ & Voltaje regulado en la salida & $200 \mathrm{~V}_{\mathrm{dc}}$ hasta $400 \mathrm{~V}_{\mathrm{dc}}$ \\
\hline $\mathrm{P}_{\text {out(max }}$ & Potencia máxima de salida & $1 \mathrm{Kw}$ hasta $2 \mathrm{Kw}$ \\
\hline $\mathrm{L}_{1}-\mathrm{L}_{2}$ & Inductores & $2 \mathrm{mH}$ \\
\hline $\mathrm{C}$ & Condensador & $2.5 \mathrm{mF}$ \\
\hline $\mathrm{f}_{\mathrm{sw}}$ & Frecuencia de conmutación & $40 \mathrm{KHz}$ \\
\hline
\end{tabular}


Los resultados de simulación con $V_{\text {in }}=169.7 V_{\text {pico, }} I=11.8 A_{\text {pico, }}, V_{0}=400 \quad V_{d c}$ y $R_{L}=160 \Omega$ demuestran el cumplimiento del balance de potencia $\left(P_{i n}=P_{0}=1 \mathrm{~kW}\right)$ en estado estable, manteniendo el voltaje de salida alrededor de $\mathrm{V}_{0}{ }^{*}=400 \mathrm{Vdc}$ con un rizado menor al $0.5 \%$; sin embargo, el análisis presentado hace énfasis en los resultados obtenidos en la entrada del convertidor donde se evidencian las ventajas de la banda de histéresis adaptativa propuesta. Las formas de onda simuladas de $\mathrm{i}_{\text {in }} \mathrm{y} \mathrm{V}_{\mathrm{in}}$ se comparan en la Fig. 5 donde $\mathrm{i}_{\text {in }}$ exhibe una forma de onda sinusoidal y en fase con $\mathrm{vin}$, alcanzando un $\mathrm{PF}=0.996$.

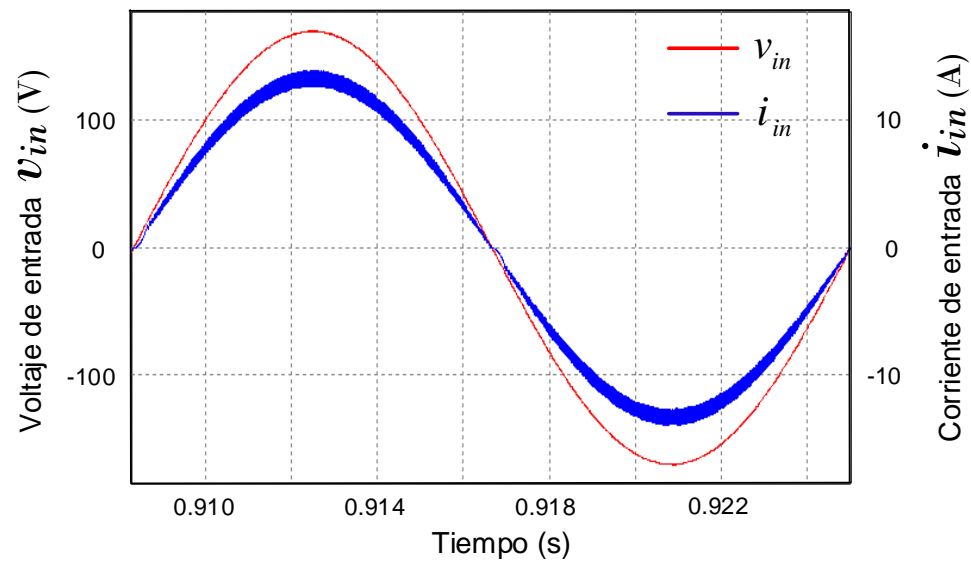

Fig. 5: Formas de onda de $\operatorname{lin} y$ vin.

La Fig. 6 muestra la forma de onda de in durante el cruce por cero. La simulación se realiza con $P_{0}=1 \mathrm{~kW}$, $V_{\text {in }}=169.7 V_{\text {pico }} y V_{\text {in }}=120 V_{\text {rms. }}$. La Fig. 6 a presenta a lin cuando el control se realiza con banda de histéresis fija, lin se deforma antes y después del cruce por cero, incrementando la $T H D_{i}$ hasta $13.3 \%$. En contraste, la Fig. $6 \mathrm{~b}$ presenta $\mathrm{i}_{\text {in }}$ cuando el control se realiza con banda de histéresis adaptativa propuesta. Esta banda de histéresis reduce la amplitud del rizado cerca del cruce por cero y el tiempo de estabilización de lin; además, la banda de histéresis adaptativa reduce la deformación en in en el cruce por cero, haciendo que lin alcance la señal de referencia por medio de una curva exponencial después del cruce por cero. Esta curva se produce debido a que la tasa de incremento de la corriente rectificada de la fuente es menor que la tasa de incremento de la señal de referencia lin $^{*}$ en los primeros $12 \mu \mathrm{s}$. EI SMC dirige a los estados del sistema para que se muevan hacía S después del cruce por cero, manteniendo cerrados Q1 y Q2. La amplitud y la duración de la curvatura tienen relación directa con el valor de $L_{1}$ y $L_{2}$.

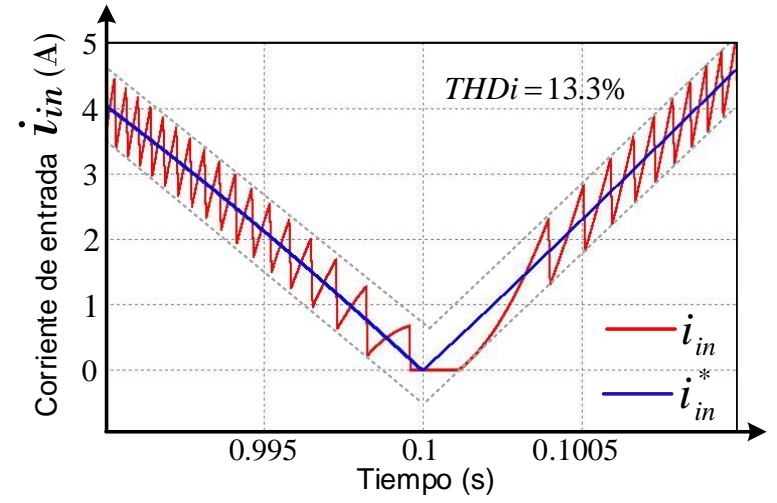

a)

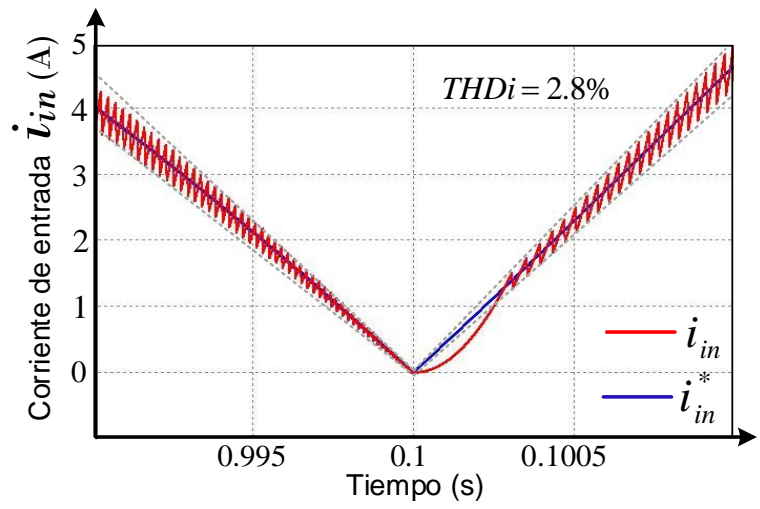

b)

Fig. 6. Forma de onda de lin durante el cruce por cero, usando: (a) banda de histéresis fija, y (b) banda de histéresis adaptativa.

La Fig. 7 muestra los resultados de la simulación del espectro en frecuencia de in. La Fig. 7a muestra el espectro con la amplitud de la banda de histéresis fija donde se puede observar una dispersión de la energía entre $60 \mathrm{~Hz}$ y $48 \mathrm{kHz}$ con THD $\mathrm{i}=13.3 \%$. La Fig. $7 \mathrm{~b}$ muestra el espectro con la banda de histéresis adaptativa propuesta donde se observa una concentración de la energía alrededor de la frecuencia fundamental de la red $(60 \mathrm{~Hz})$ y de la frecuencia de conmutación constante $(40 \mathrm{kHz})$; razón por la cual, el THDi se reduce hasta 2.8\% manteniéndolo por debajo de los límites máximos permisibles del estándar EN/ IEC 61000-3-2 (1000-3-2).

El convertidor SBLB PFC es simulado con un incremento de carga del $200 \%$ Para ilustrar la efectividad del SMC con la banda de histéresis propuesta para controlar la dinámica del sistema. La Fig. 8 muestra los resultados de la simulación cuando $P_{0}$ se incrementa desde $1 \mathrm{~kW}$ hasta $2 \mathrm{~kW}$, manteniendo la amplitud de, vin constante. 
lin se mantiene sinusoidal y en fase con vin, garantizando la corrección del PF. El THDi se mantiene constante en $2.8 \%$ durante el tiempo de simulación. El controlador con la banda de histéresis adaptativa propuesta requiere $\sim 87 \mathrm{~ms}$ (aproximadamente 5 ciclos de la red) para alcanzar la corriente de referencia necesaria para suministrar los $2 \mathrm{~kW}$ en la carga. El voltaje en la salida se estabiliza en $~ 345 \mathrm{~ms}$ (aproximadamente 21 ciclos de la red). La simulación muestra que el controlador con la banda de histéresis adaptativa propuesta permite reducir el $T H D_{i}$, la deformación de la corriente en el cruce por cero y corregir el PF en presencia de cambios significativos de la región de operación.
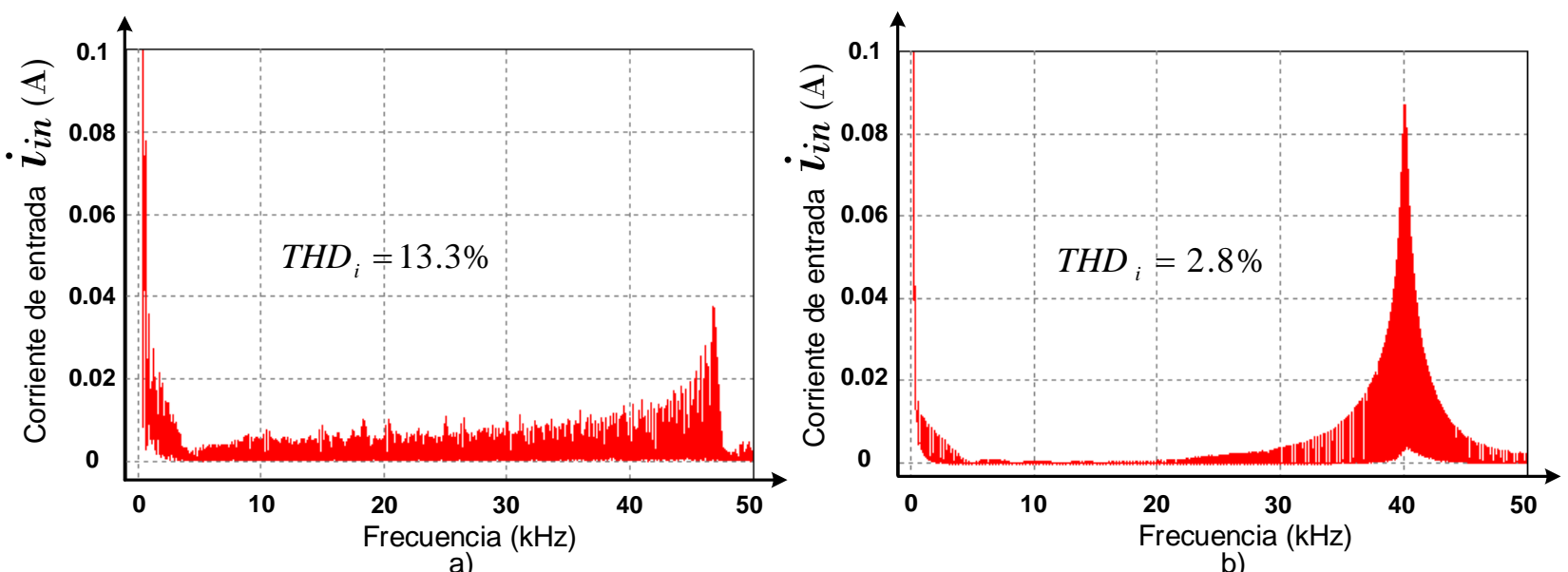

Fig. 7: Espectro de frecuencias de la corriente lin: (a) banda de histéresis fija y (b) banda de histéresis adaptativa.

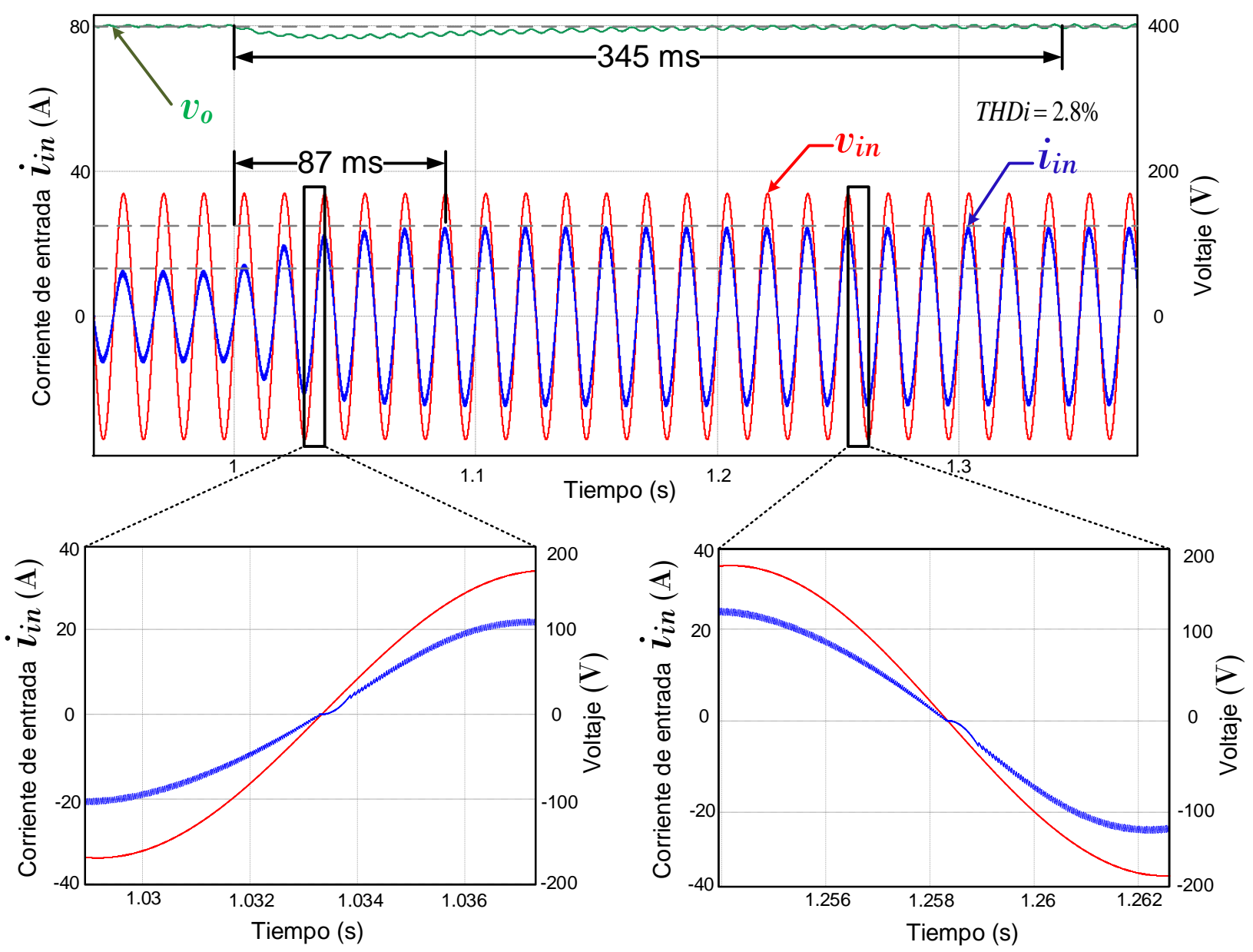

Fig. 8: Respuesta transitoria cuando la carga se incrementa desde $1 \mathrm{~kW}$ hasta $2 \mathrm{~kW}$. La corriente se estabiliza aproximadamente en 5 ciclos de la red. 


\section{CONCLUSIONES}

La banda de histéresis adaptativa propuesta en este artículo permite reducir el rizado, el $T H D_{i}$ y fijar la frecuencia de conmutación del SBLB PFC. Los resultados muestran que la señal de corriente en la entrada se mantiene en fase con el voltaje, garantizando un factor de potencia de 0.994 . Además, la deformación de la corriente durante el cruce por cero de la señal es reducida en comparación con la deformación producida por la banda de histéresis de amplitud constante. El espectro en frecuencia de la señal de la corriente de entrada muestra la concentración de la energía alrededor de la frecuencia fundamental de la red y de la frecuencia de conmutación de $40 \mathrm{kHz}$ del SBLB PFC cuando se usa la banda de histéresis adaptativa; en consecuencia, el THD i es reducido de acuerdo al estándar EN/ IEC 61000-3-2 (1000-3-2).

La banda de histéresis adaptativa propuesta permite alcanzar los objetivos de control, evitando afectar la robustez, la regulación y la respuesta dinámica del SMC para todas las condiciones simuladas. EI THD i es reducido y el PF aumenta en presencia de cambios de carga de hasta el $200 \%$, manteniendo la frecuencia de conmutación constante y la deformación de la señal baja en el cruce por cero.

\section{AGRADECIMIENTOS}

Los autores agradecen a la Corporación Universitaria Minuto de Dios UNIMINUTO y a la Universidad de Antioquia (UdeA) por el apoyo recibido mediante la estrategia de sostenibilidad.

\section{REFERENCIAS}

Alam, M., W. Eberle, D.S Gautam, C. Botting, N. Dohmeier y F. Musavi, A hybrid resonant pulse-width modulation bridgeless AC-DC power factor correction converter, IEEE Transactions on Industry Applications, 53(2), 1406-1415 (2017)

Alsmadi, U.V., M.A. Haj-ahmed y L. Xu, Sliding mode control of power converters: DC/DC converters, International Journal of Control, 91(11), 2472-2493 (2017)

Ashita, S., G. Uma y P. Deivasundari, Chaotic dynamics of a zero average dynamics controlled DC-DC Ćuk converter, IET Power Electronics, 7(2), 289-298 (2014)

Bin, K.S y D. Lu, ZCS Bridgeless Boost PFC Rectifier Using Only Two Active Switches, IEEE Transactions on Industrial Electronics, 62(5), 2795-2806 (2015)

Chen, H.C., C.Y. Lu, G.T. Li y W.C. Chen, Digital Current Sensorless Control for Dual-Boost Half-Bridge PFC Converter With Natural Capacitor Voltage Balancing, IEEE Transactions on Power Electronics, 32(5), 4074-4083 (2017)

Chih-Chiang, H., F. Yi-Hsiung y H. Chin-Hsiung, Zero-voltage-transition bridgeless power factor correction rectifier with soft-switched auxiliary circuit, IET Power Electronics, 9(3), 546-552 (2016)

Chincholkar, S.H y C.Y. Chan, Design of Fixed-Frequency Pulsewidth-Modulation-Based Sliding-Mode Controllers for the Quadratic Boost Converter, IEEE Transactions on Circuits and Systems II: Express Briefs, 64(1), $51-55$ (2017)

Franceschini, G., E. Lorenzani y G. Buticchi, Saturation compensation strategy for grid connected converters based on line frequency transformers, IEEE Transactions on Energy Conversion, 27(2), 229-237 (2012)

Guzman, R., L. Garcia, J. Morales, M. Castilla y J. Matas, Sliding-Mode Control for a Three-Phase Unity Power Factor Rectifier Operating at Fixed Switching Frequency, IEEE Transactions on Power Electronics, 31(1), 758-769 (2016)

Huang, L., F. Chen, W. Yao y Z. Lu, Flexible Mode Bridgeless Boost PFC Rectifier with High Efficiency over a Wide Range of Input Voltage, IEEE Transactions on Power Electronics, 32(5), 3513-3524 (2017)

Huber, L., Y. Jang y M. Jovanovic, Performance Evaluation of Bridgeless PFC Boost Rectifiers, IEEE Transactions on Power Electronics, 23(3), 1381-1390 (2008)

Jovanovic, M.M e Y. Jang, State-of-the-art, single-phase, active power-factor-correction techniques for high-power applications - an overview, IEEE Transactions on Industrial Electronics, 52(3), 701-708 (2005)

Kim, Y., W. Sung y B. Lee, Comparative Performance Analysis of High Density and Efficiency PFC Topologies, IEEE Transactions on Power Electronics, 29(6), 2666-2679 (2014)

Man, C.N., R. Tin-ho y K. King-Man, Active Virtual Ground - Bridgeless PFC Topology, IEEE Transactions on Power Electronics, 32(8), 6206-6218 (2017)

Marcos-Pastor, A., E. Vidal-Idiarte, A. Cid-Pastor y L. Martinez-Salamero, Interleaved Digital Power Factor Correction Based on the Sliding-Mode Approach, IEEE Transactions on Power Electronics, 31(6), 4641-4653 (2016)

Marcos-Pastor, A., E. Vidal-Idiarte, A. Cid-Pastor y L. Martinez-Salamero, Loss-Free Resistor-Based Power Factor Correction Using a Semi-Bridgeless Boost Rectifier in Sliding-Mode Control, IEEE Transactions on Power Electronics, 30(10), 5842-5853 (2015)

Mejía-Ruiz, G., N. Muñoz-Galeano y J. Lopez-Lezama, Modeling and development of a bridgeless PFC Boost rectifier, Revista Facultad de Ingeniería, 82, 9-21 (2017) 
Mohanty, P.R y A.K. Panda, Fixed-Frequency Sliding-Mode Control Scheme Based on Current Control Manifold for Improved Dynamic Performance of Boost PFC Converter, IEEE Journal of Emerging and Selected Topics in Power Electronics, 5(1), 576-586 (2017)

Muñoz-Galeano, N., J.B. Cano-Quintero y J.M. López-Lezama, Control de un Convertidor Elevador de Voltaje AC/DC Utilizando Balance de Potencias, doi.org/10.4067/S0718-07642016000200002, Información Tecnológica, 27(2), 3-10 (2016)

Musavi, F., W. Eberle y W.G. Dunford, A Phase-Shifted Gating Technique With Simplified Current Sensing for the SemiBridgeless AC-DC Converter, IEEE Transactions On Vehicular Technology, 62(4), 1568-1576 (2013)

Pichan, M. y H. Rastegar, Sliding-Mode Control of Four-Leg Inverter With Fixed Switching Frequency for Uninterruptible Power Supply Applications, IEEE Transactions on Industrial Electronics, 64(8), 6805-6814 (2017)

Repecho, V., D. Biel, J.M. Olm y E.F. Colet., Switching Frequency Regulation in Sliding Mode Control by a Hysteresis Band Controller, IEEE Transactions on Power Electronics, 32(2), 1557-1569 (2017)

Utkin, V., Variable structure systems with sliding modes, IEEE Transactions on Automatic Control, 22(2), 212-222 (1977)

Utkin, V., Discussion Aspects of High-Order Sliding Mode Control, IEEE Transactions on Automatic Control, 61(3), 829$833(2016)$

Utkin, V., J. Guldner y J. Shi, Sliding Mode Control in Electro-Mechanical Systems, $2^{\text {nd }}$ Ed., 237, CRC Press, Boca Ratón, EUA (2009)

Xu, H., W. Li, H. Zhang, X. Hao e Y. Zhang, Bridgeless pfc power converter with high efficiency, Google Patents, US14262660 (2014)

Ye, J., P. Malysz y A. Emadi, A fixed-switching-frequency integral sliding mode current controller for switched reluctance motor drives, IEEE Journal of Emerging and Selected Topics in Power Electronics, 3(2), 381-394 (2014)

Yonglu, L., S. Yao y S. Mei, A Control Method for Bridgeless Cuk/Sepic PFC Rectifier to Achieve Power Decoupling, IEEE Transactions on Industrial Electronics, 64(9), 7272-7276 (2017)

Young, K.D., V.I. Utkin y U. Ozguner, A control engineer's guide to sliding mode control, IEEE Transactions on Control Systems Technology, 7(3), 328-342 (1999) 